\title{
A rare presentation of a retroperitoneal cystic teratoma in an adult.
}

\author{
Elroy Weledji ${ }^{1}$ and Ngwane Ntongwetape ${ }^{2}$ \\ ${ }^{1}$ University of Buea \\ ${ }^{2}$ Affiliation not available
}

January 21, 2021

\begin{abstract}
Being of embryonal tissue origin, the teratomas are rare in adults, typically occurring either in the gonads or in the sacrococcygeal region in infancy and childhood. We present a case of a teratoma in the lesser sac of an adult female managed by en-bloc resection with the infiltrating stomach segment.
\end{abstract}

Clinical Image

Title: A rare presentation of a retroperitoneal cystic teratoma in an adult.

${ }^{1}$ Elroy Patrick Weledji, ${ }^{2}$ Ngwane Ntonwetape

${ }^{1}$ Department of Surgery, Faculty of Health Sciences, University of Buea, Cameroon

${ }^{1}$ Regional Hospital Limbe, Limbe, S.W.Region, Cameroon

${ }^{2}$ Regional Hospital Buea, Buea, S.W. Region Cameroon

${ }^{1}$ Corresponding author: E.P. Weledji. PO Box 126, Limbe, S.W. Region, Cameroon. Email:elroypat@yahoo.co.uk; Tel:237699922144

\section{Abstract:}

Being of embryonal tissue origin, the teratomas are rare in adults, typically occurring either in the gonads or in the sacrococcygeal region in infancy and childhood. We present a case of a teratoma in the lesser sac of an adult female managed by en-bloc resection with the infiltrating stomach segment.

\section{Clinical message :}

Retroperitoneal teratomas are rare in adults and, present challenging surgical management as they usually infiltrate into adjacent retroperitoneal organs. Most are benign but the malignant mature cystic teratomas have the potential to metastasize to retroperitoneal lymph nodes and lung parenchyma. The definitive treatment of these neoplasms is surgical resection.

\section{Case:}

A 27-yr-old African woman presented with a 2 year history of an abdominal mass that waned in size. It was associated with abdominal bloatedness, postpandrial vomiting, lethargy and difficulty in performing her daily activities. On examination she was clinically well but exhausted from the weight of the abdominal mass. Physical examination revealed a large circumscribed mobile abdominal mass of $\sim 20 \mathrm{~cm} d$, extending across the upper abdomen that did not move with respiration. There were no hepatosplenomegaly nor ascites. Ultrasonography suggested a retroperitoneal mesenteric mass with no lymphadenopathy. Blood tests were within the normal range. Laparotomy revealed a circumscribed retroperitoneal mass in the 
stomach bed protruding into the lesser sac and adherent to the transverse colon, mesocolon and posterior surface of stomach with dilated gastroepiploic veins. There was mucinous exudate from the cystic mass but no evidence of metastases. A difficult mobilization of the mass allowed entry into the lesser sac. There was no cleavage plane between the posterior stomach wall and thus the posterior stomach wall was excised en bloc with the mass (figure 1). The stomach defect was closed and the mesenteric defect closed to prevent an internal hernia. Postoperative recovery was unremarkable. Histology confirmed a benign cystic teratoma (figure 2).

\section{References}

1. Liu H, Wanmeng L, Wenlong Y, Youfei Q. Giant retroperitoneal teratoma in adult. Am J Surg 2007;193(6):736-737

2. Gatcombe HG, Assikis V, Kooby D, Johnstone PAS. Primary retroperitoneal teratomas: a review of the literature. J Surg Oncol 2004;86(2): 107-113

3. Pinson CW, Remine SG, Fletcher WS, Braasch JW. Long-term results with primary retroperitoneal tumors. Archives of Surgery 1989; 124(10):1168-1173

\section{Figures}

Figure 1. Cystic teratoma $(15 \mathrm{~kg}, 15 \mathrm{cmx} 10 \mathrm{cmx} 6 \mathrm{~cm})$ Figure 2: Cystic teratoma composed of somatic cell types from two or more embroyonic germ layers ( ectoderm, mesoderm or endoderm) 